\title{
ILLUSTRATING
}

A THEMED REVIEW OF

THE EXPERIENCE OF CHILDREN \&

YOUNG PEOPLE CARED FOR IN

MENTAL HEALTH, LEARNING DISABILITIES,

\& AUTISM INPATIENT SETTINGS

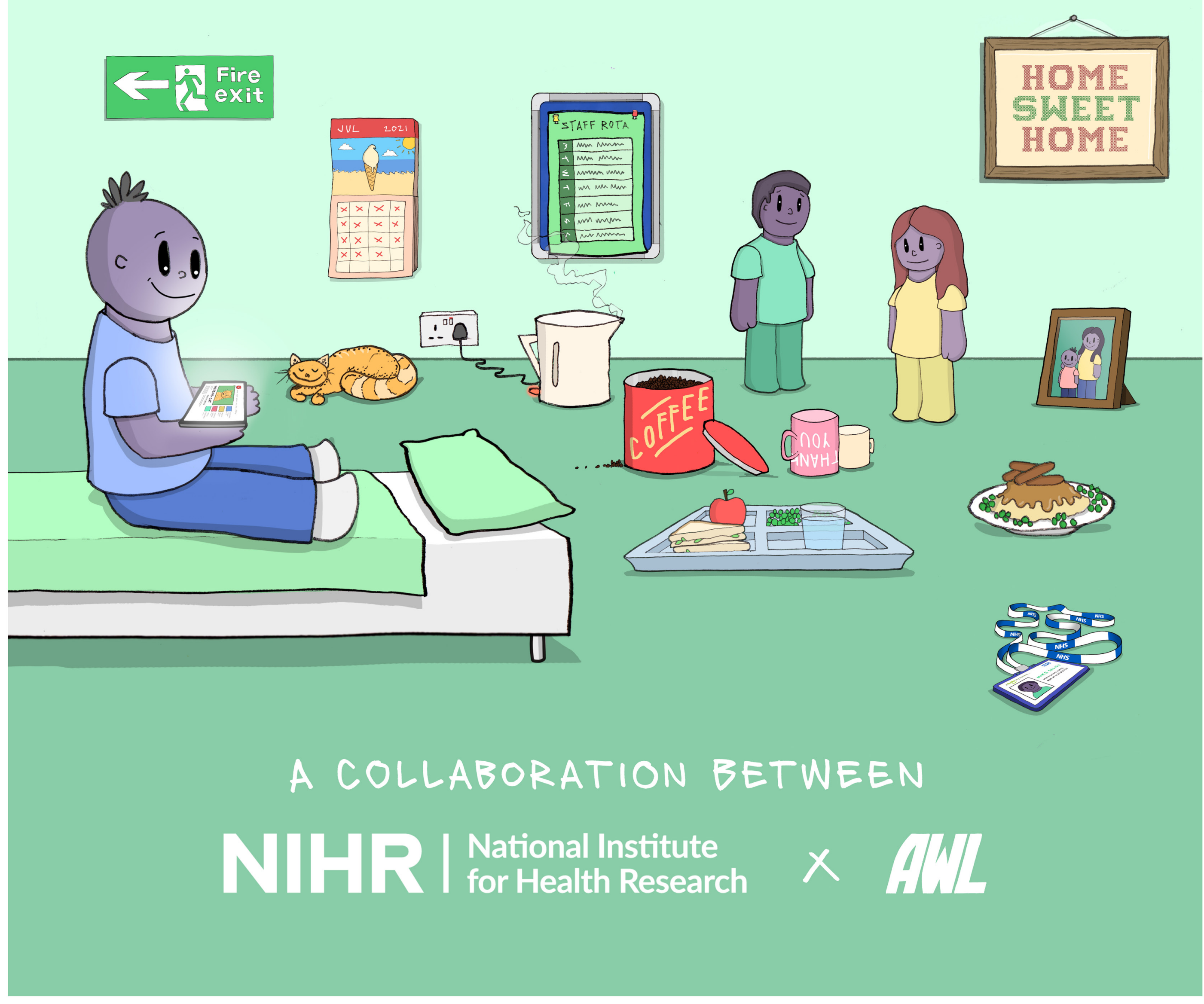




\section{The experience of children and young people cared for in mental health, learning disability, and autism inpatient settings}

For the first time, working in collaboration with the art collective Act With Love, the National Institute for Health Research Centre for Engagement and Dissemination (NIHR CED)* has presented key messages from a Themed Review as illustrations. We hope that presenting our findings in this way will make them more accessible and engaging so that they reach more people and have a greater impact.

The Themed Review focuses on the experience of children and young people cared for in mental health, learning disability, and autism inpatient settings. It brings together both academic study and practical wisdom from lived experience. As well as searching the scientific literature, we consulted children and young people, their parents, and staff, to see how far the evidence reflected their experiences. We also collected online resources including first person accounts and audits. This review combines these different strands of evidence to make sense of current knowledge and practice and to suggest opportunities for service improvement.

Through careful consideration and collaboration, we have brought the key findings of the review to life by working personal experiences and evidence into illustrations. The cartoon nature of the images is not intended to demean the importance or sensitivity of the subject matter, instead it is an attempt to make the key messages more accessible and to provoke thought and conversation. Our hope is that these illustrations represent those with experience of these settings and help to educate those without this experience.

We are continually trying to better understand the views and needs of our audiences, and make improvements in the way we share knowledge. We would love to hear your thoughts and reactions to this style of dissemination so, if you have time, please share your thoughts with us by completing our brief online survey.

\section{Candace Imison}

Associate Director of Evidence and

Dissemination (NIHR CED)
Jodi Brown

Senior Stakeholder Engagement

Manager (NIHR CED)
\#CYPMentalHealth
@NIHREvidence

* The NIHR Centre for Engagement and Dissemination (NIHR CED) shares knowledge and promotes in-depth discussion with the aim of improving health and social care. 


\section{Good relationships}

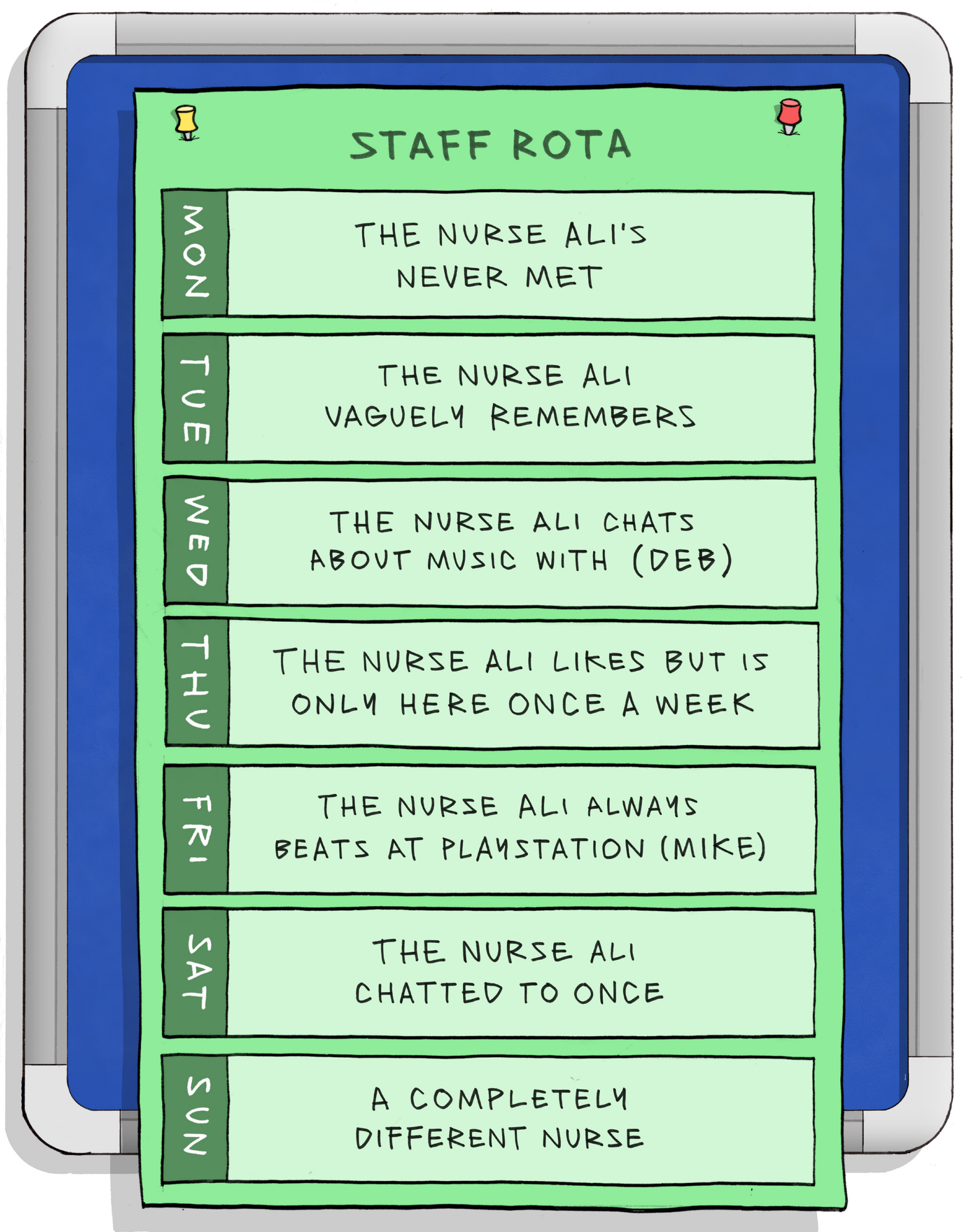

An inpatient admission creates a new set of relationships between a young person, their family, and the staff on the ward. Staff need to have enough time to develop meaningful relationships with the young people they are looking after. Trusting and empathetic acts of care, outside of therapeutic sessions, can improve the quality of a young person's experience and wellbeing. 


\section{Normality}
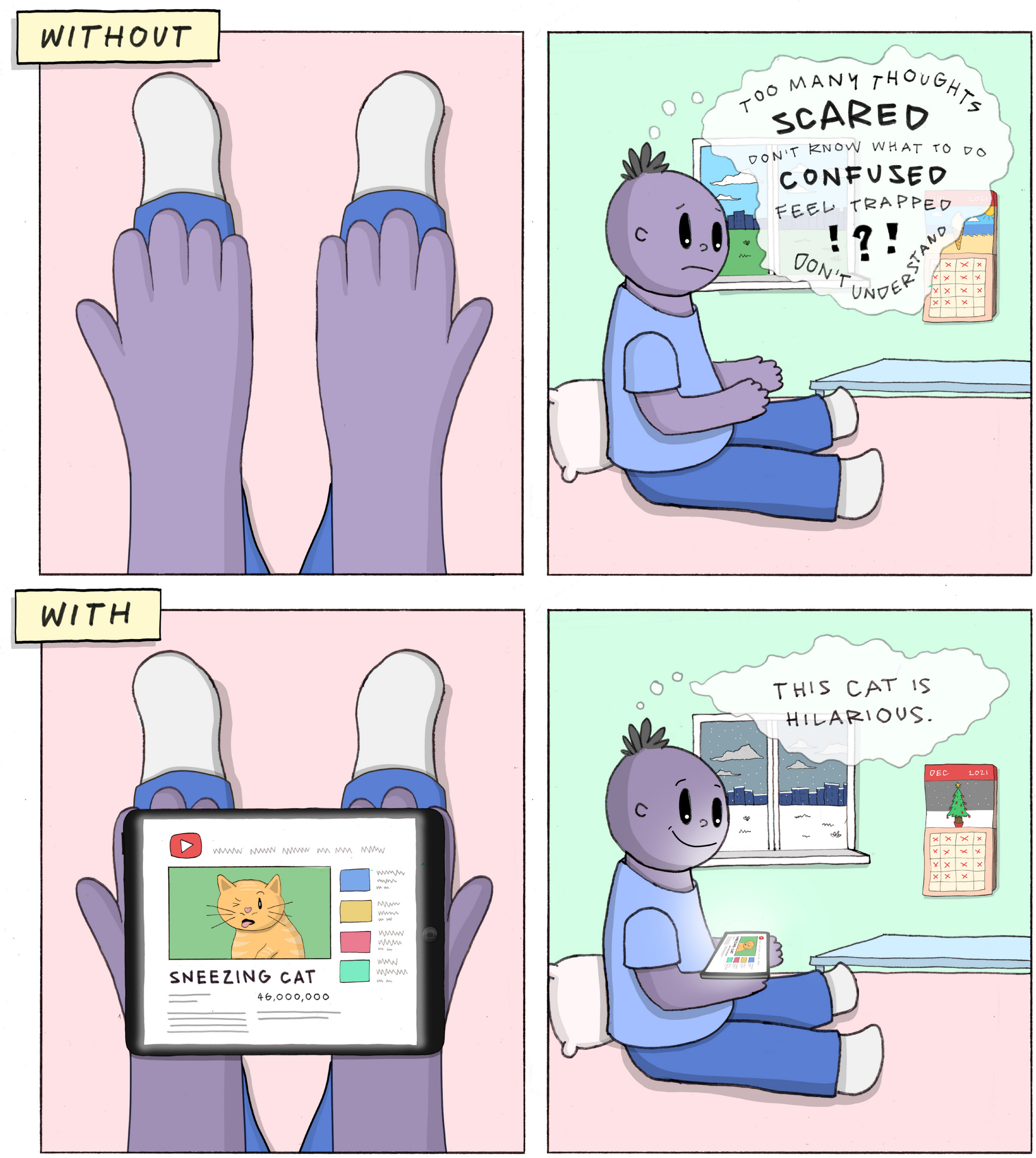

Lengthy admissions can dislocate a young person from the things they value most in their day-to-day lives.

Inpatient settings may lack the most important aspects of 'homeliness', especially if there are rules or 'blanket restrictions' that do not take into account individual needs. Promoting a sense of normality wherever possible, through flexibility and personalised care, can significantly

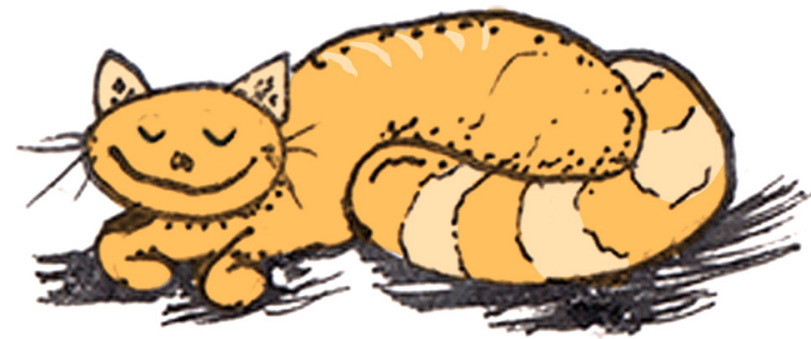
improve a young person's experience and wellbeing. 


\section{Restrictive practise}
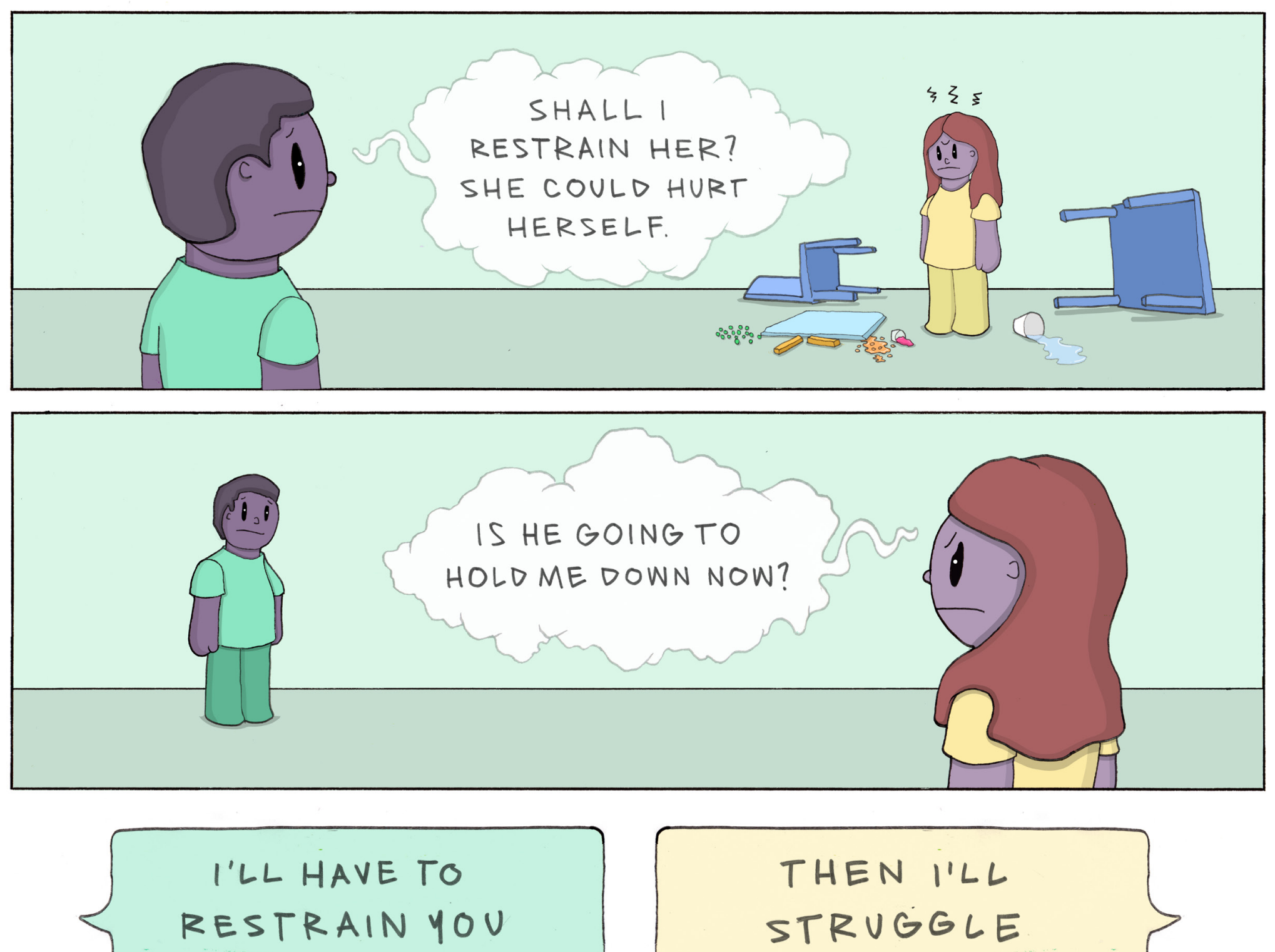

THEN I'LL

STRUGGLE

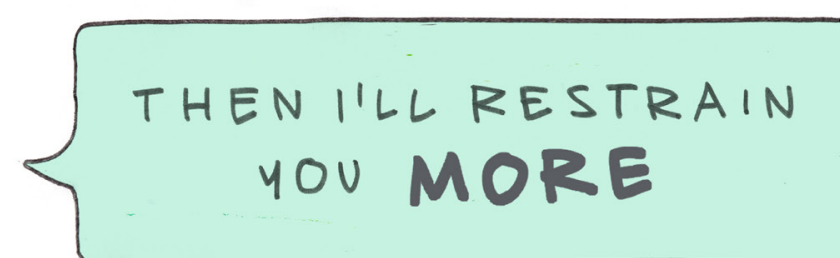

THEN I'LL STRUGGLE MORE

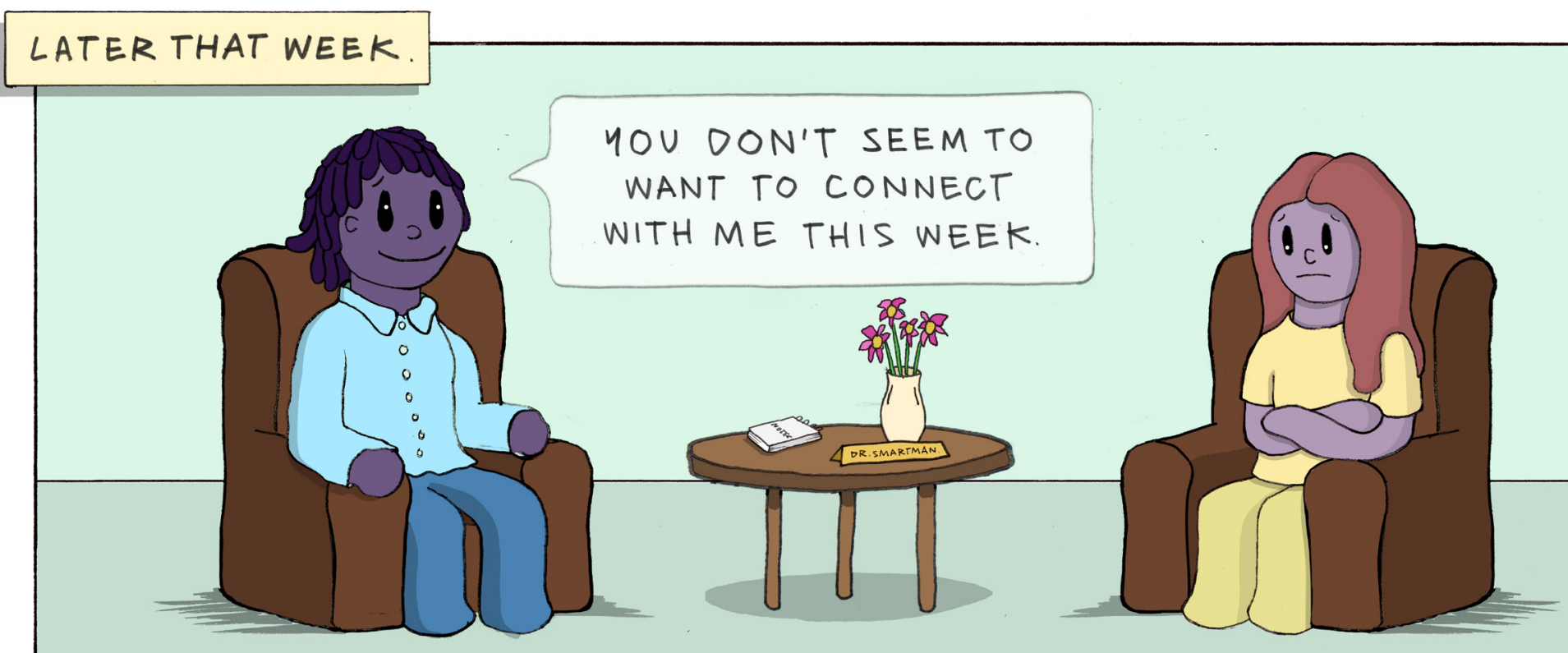

Physical restraint can have negative consequences for all involved and should only be used as a last resort, to prevent harm to a young person or member of staff. It can leave the young person feeling scared and may trigger memories of past trauma. This can damage therapeutic relationships and mean a young person is less likely to engage in treatment. Staff may feel guilt and regret, even when they believe restraint was necessary. High-quality staff training (adopting Restraint Reduction Network standards) and good, needs-based, care planning can avoid unnecessary use of restraint. 


\section{It isn't easy for anyone}

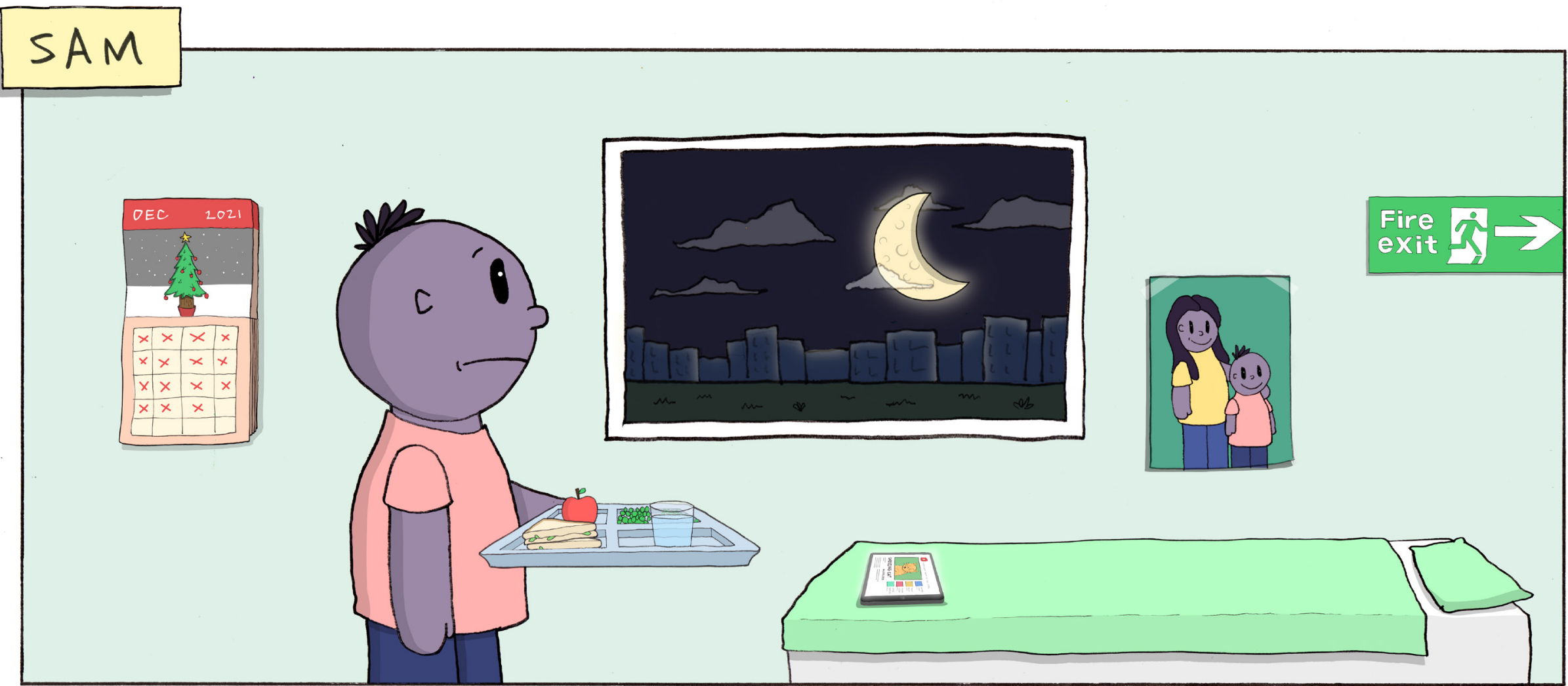

\section{SAM'S NURSE}
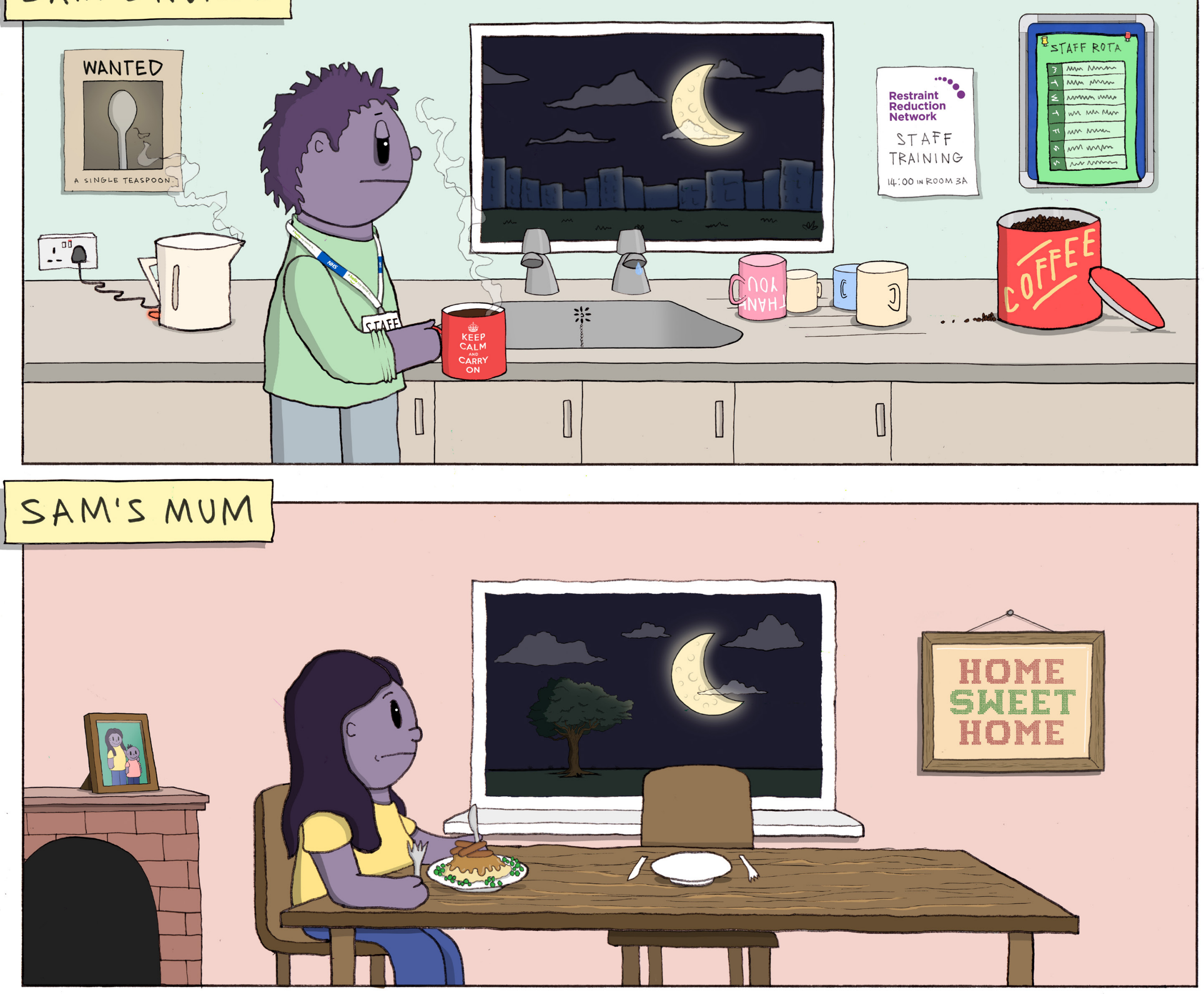

An inpatient admission is not easy for anyone involved: the young person, their family, carers, and hospital staff. Meeting everyone's needs can be challenging. Understanding and respecting everyone's perspective is essential to improve experience and outcomes. 


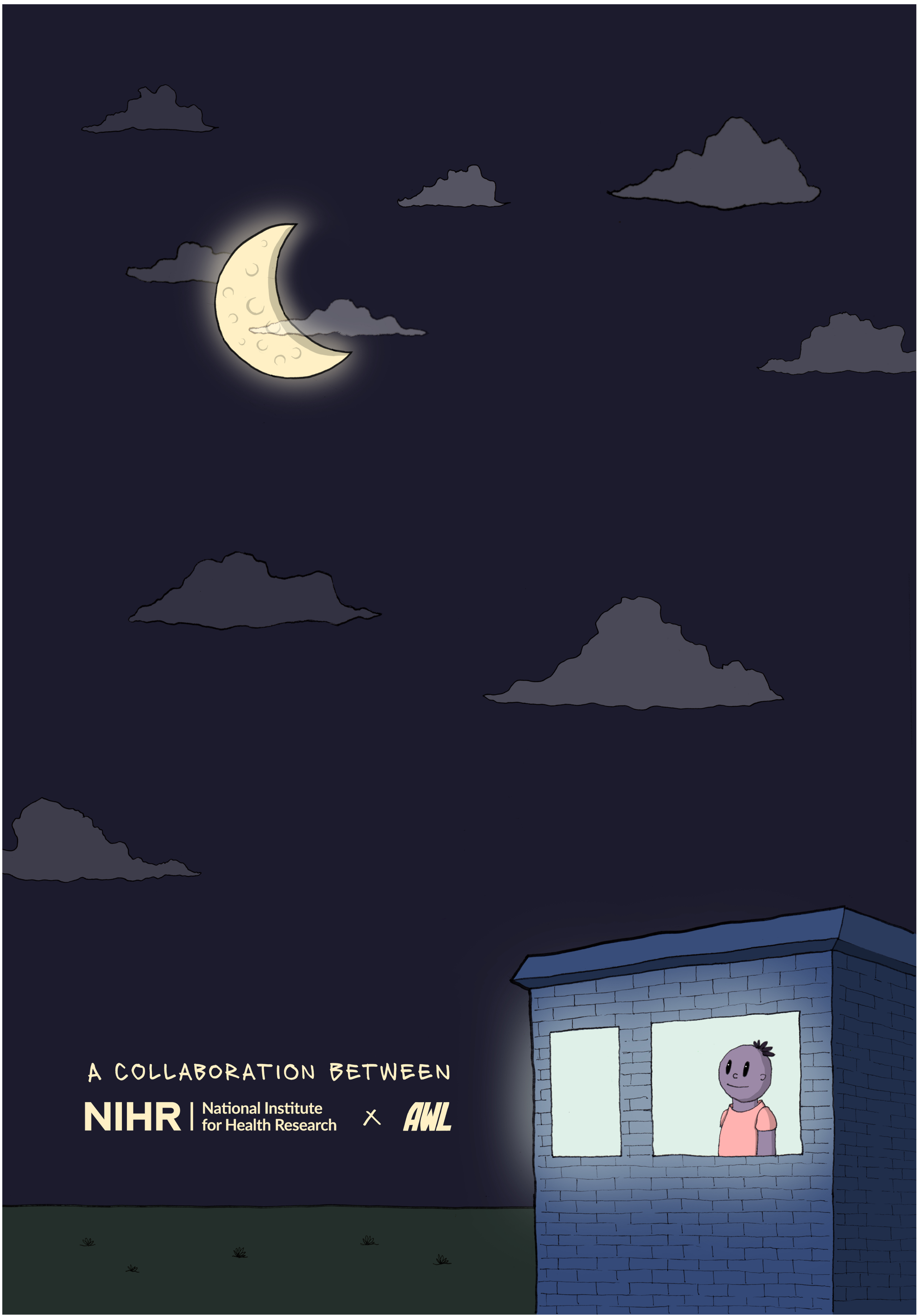

